\title{
ESTERILIDAD INDIVIDUAL EN RHODNIUS PROLIXUS STAL. TRATADOS CON METEPA (OXIDO DE TRIS (1-(2 METIL) AZIRIDINIL) FOSFURO), $Y$ ACCION DEL MISMO SOBRE TRYPANOSOMA CRUZI
}

\author{
M. Dora FELICIANGELI * \\ Rosa de HÜBSCH * \\ Norma de CHIECHI *
}

\section{RSPSP-118}

Feliciangeli, M. D. et al. - Esterilidad individual en Rhodnius prolixus Stal. tratados con metepa (oxido de tris (1-(2 metil) aziridinil) fosfuro), $y$ accion del mismo sobre Trypanosoma cruzi. Rev. Saúde públ., S Paulo, 6:79-83, 1972.

Resumen: Se analizan los factores que interfieren en la esterilidad inducida quimicamentie en R. Prolixus mediante aplicaciones topicas en distintas dosis de metepa. Se sugiere profundizar el estudio de la susceptibilidad individual para conocer la composición de la población frente al quimioesterilizante y la probable aparición de resistencia. Se investiga además la acción del metepa sobre $\mathrm{T}$. cruzi en triatominos infiectados experimentalmente, obteniéndose resultados negativos.

UNITERMinos: Rhodnius prolixus*; Esterilidad inducida*; Metepa; Trypanosoma cruzi.

\section{N T RODUCCION}

El estudio de un nuevo método de control para los vectores de la Enfermedad de Chagas en Venezuela se ha hecho necesario con la aparición de una cepa de
Rhodnius prolixus resistente al dieldrin en el Edo. Trujillo**.

Durante la última década los esterili zantes químicos han sido estudiados como un arma promisora contra los in. sectos nocivos para la salud, sugiriendo LINDQUisT ${ }^{\circ}$ la posibilidad de acelerar el control de las enfermedades metaxénicas interfiriendo por una parte en la fertilidad de los vectores, y por otra en el desarrollo de los agentes patógenos de los cuales son portadores.

Los experimentos de laboratorio con metepa han demostrado que este producto es eficaz para $R$. prolixus, lográndose en los machos, con $100 \mu \mathrm{g} /$ individuo, aproximadamente el 90\% de esterilidad, sin que fueran afectados la longevidad $y$ el comportamiento de los insectos 3 .

En el presente trabajo se estudian las variaciones individuales de la fertilidad, tratando de analizar los factores que sobre ella influyen, y la acción del metepa sobre Trypanosoma cruzi en triatominos infectados experimentalmente, con la $\mathrm{fi}$ nalidad de investigar la possibilidad de una doble acción del quimioesterilizante. \footnotetext{
* De la Dirección de Malariologia y Saneamiento Ambiental, División de Endemias Rurales,
}

* Gomizz - NuTNEz, J, C. - Infromación verbal. 
FELICIANGELI, M. D. et al. - Esterilidad individual en Rhodnius prolixus Stal. tratados con metepa (oxido de tris (1-(2 metil) aziridinil) fosfuro), y accion del mismo sobre Trypanosoma cruzi. Rev. Saúde públ., S. Paulo, 6:79-83, 1972.

\section{MATERIAL Y METODO}

Para los experimentos se utilizaron $R$. prolixus provenientes de una colonia del laboratorio mantenidas en condiciones standard 4, de la misma edad y condiciones morfo-fisiológicas: 7-10 días de adultos, en ayunas y separados por sexo durante el $\mathrm{V}$. $^{\circ}$ estadio ninfal.

Para la esterilización fué usado metepa el $82 \%$ disuelto en acetona de las concentraciones: $2,5 \% ; 5 \% ; 10 \%$ y $20 \%$. La aplicación tópica sobre el tórax de los insectos anestesiados con $\mathrm{CO}_{2}$, se hizo usando una microjeringa calibrada anexa a micrómetro de mano con $2 \mu$ l. de solución, suministrándose por lo tanto dosis individuales de: 50; 100; 200 y 400 $\mu \mathrm{g}$ respectivamente.

Cada concentración fue ensayada sobre 10 machos y 10 hembras, cada uno de los cuales fue aislado en un frasco pequeño de vidrio, apareado con un individuo normal del sexo opuesto y alimentado. A los 14 días se recogieron los huevos y se eliminaron los insectos.

Para estudiar la acción del metepa sobre T. cruzi se utilizó un grupo de 100 machos tratados con $100 \mu \mathrm{g} / \mathrm{cada}$ uno, $\mathrm{y}$ dos grupos testigos, uno constituído por 100 insectos normales $y$ el otro por 100 insectos tratados con el solvente solamente.

La infección de los tratominos se realizó alimentándolos de ratones blancos, de la cepa MRT altamente infectados con $T$. cruzi ( 3 a 4 cruces de parasitemia) de la cepa E.P. procedente de la Universidad de Carabobo aislada de humano y mantenida en el laboratorio a través de repiques en ratones desde el año 1967. Para la alimentación se utiliz6 un ratón por cada 10 insectos. Los ratones fueron inmovilizados en pequeñas jaulas metálicas (Fig. 1) y colocados con los 10 insectos en un recipiente de vidrio. El tiempo mínimo de alimentación

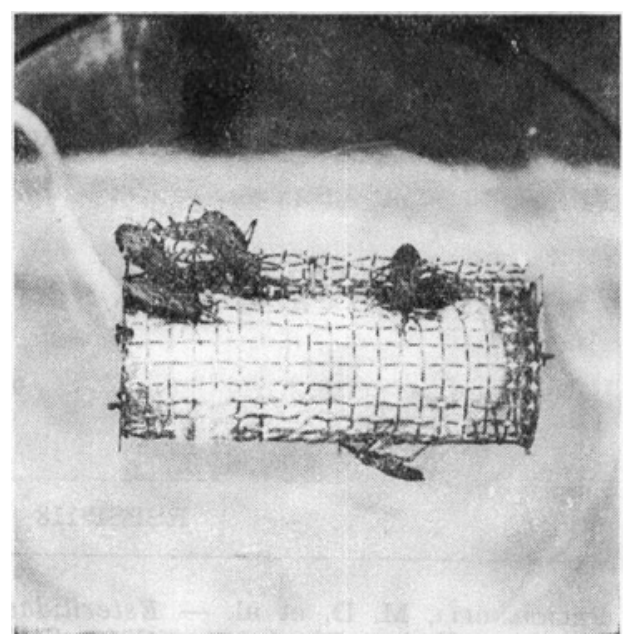

Fig. $1-R$. prolixus adultos alimentándose sobre ratón blanco infectado por $T$. cruzi.

fué de 30 minutos, pero se esperó hasta que todos los insectos se hubieran alimentado. El examen de heces de los triatominos se hizo según la técnica descrita por DIAZ ${ }^{2}$.

El primer examen se efectuó a los 20 días después de la primera comida: los triatominos positivos fueron siempre eliminados y los negativos se siguieron alimentando y examinando rutinariamente cada 14 días hasta la muerte.

\section{RESULTADOS}

Fertilidad: La Figura 2 representa el $\%$ de eclosión de huevos de las $ㅇ$ nor. males apareadas con los machos trata. dos.

La Figura 3 representa la fertilidad relativa en las hembras tratadas en las cuales, por efecto del quimioesterilizante, hay una disminución en la producción de huevos, directamente proporcional a la dosis suministrada, por 10 tanto refiriendo este valor al testigo, según morjficación de la fórmula de Toppozada 8: 
FELICIANGELI, M. D. et al. - Esterilidad individual en Rhodnius prolixus Stal. tratados con metepa (oxido de tris (1-(2 metil) aziridinil) fosfuro), y accion del mismo sobre Trypanosoma cruzi. Rev. Saúde públ., S. Paulo, 6:79-83, 1972,

$$
\text { Fertilidad relativa }=\frac{\% \text { eclosión tratados } \times \mathrm{N} \cdot{ }^{\circ} \text { huevos } / q \text { tratadas }}{\% \text { eclosión testigo } \times \mathrm{N} \cdot \cdot^{\circ} \text { huevos } / q \text { testigo }}
$$

Cada barra representa el valor medio y los valores mínimo y máximo que se obtuvieron en el grupo.

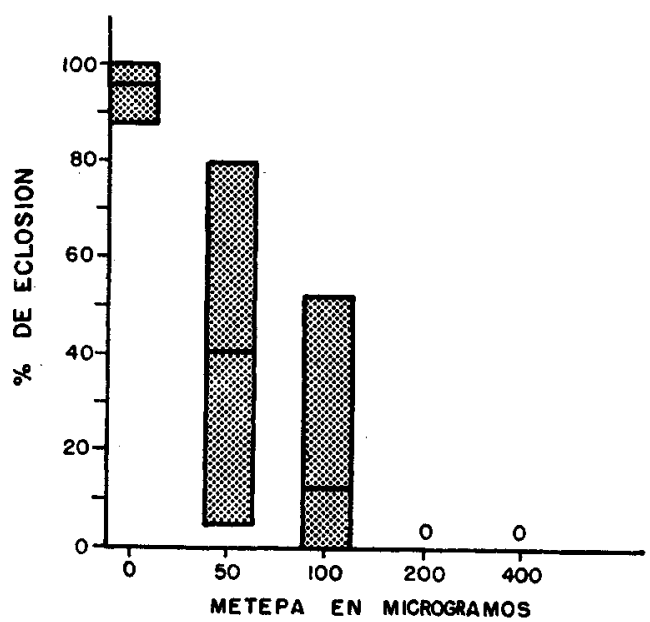

Fig. 2 - Media aritmética y rangos de eclosión en huevos de $Q \underline{q}$ normales apareadas con $\delta^{x} \sigma^{x}$ tratados con distintas dosis de metepa.

En la Figura 2 se nota que el grupo testigo fué bastante homogéneo, siendo el extremo superior $100 \%$ y el inferior $88,2 \%$, con una $\bar{x}=95,7 \pm 9,4$ (Media $\pm \mathrm{DE})$.

Cuando se trataron los machos con $50 \mu \mathrm{g}$., los \% de eclosión variaron marcadamente, abarcando valores desde el $78,9 \%$ hasta $4,6 \%$ siendo $\bar{x}=40.4 \pm 21$, distribuyéndose los valores en propor ción casi igual a un lado y otro de la media.

El \% de eclosión de huevos de las hem. bras que se aparearon con los machos tratados con $100 \mu \mathrm{g}$. fué $\mathrm{x}^{-}=12,2 \pm$ 19,8 , agrupándose el $70 \%$ de los valores hacia el $0 \%$ y el $\mathbf{3 0} \%$ hacia el $\mathbf{5 2 , 0} \%$.

Con $200 \mu \mathrm{g}$. y $400 \mathrm{~m} \mu \mathrm{g}$. se obtuvo la esterilización completa en los machos.
En la Figura 3, para el grupo testigo aparece un valor de fertilidad muy bajo, del $66,6 \%$. En el grupo tratado con $50 \mu \mathrm{g}$. no se registró mucha variación y se nota que la eficacia del metepa sobre las hembras a esta dosis es muy escassa. Con $100 \mu \mathrm{g}$. los valores vuelven a dispersarse alrededor de la $\bar{x}=44,5 \pm 26,1$ con un $60 \%$ por encima y el $40 \%$ por debajo de la misma.

En los grupos tratados con $200 \mu \mathrm{g}$. y $400 \mu \mathrm{g}$. la acción es casi la misma: ambas barras llegan a un valor máximo del $27,0 \%$ y el mínimo del $0 \%$, disminuyendo ligeramente la $\bar{x}$ de $18,1 \pm 6,8$ a $15,5 \pm$ 7,0 para la dosis mayor.

Acción del metepa sobre T. cruzi.

La mortalidad ocurrida sobre los insectos en experimento redujo el $\mathrm{N} .^{\circ}$ inicial de 300 a 236, examinándose 72 triatominos sin tratar, 94 tratados con el solvente y 70 tratados con metepa. En-

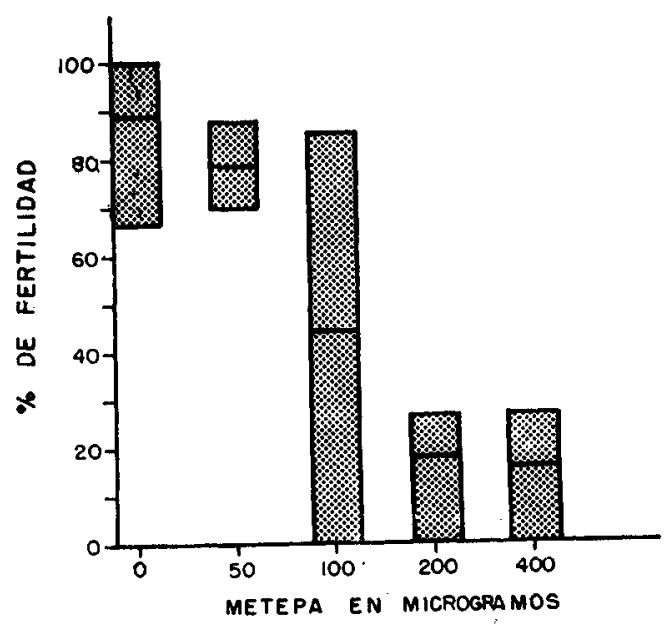

Fig. 3 - Media aritmética y rangos de fertilldad en $Q Q$ tratadas con distintas dosis de metepa, $y$ apareadas con $\delta \delta$ normales. 
FELICIANGELI, M. D. et al. - Esterilidad individual en Rhodnius prolixus Stal. tratados con metepa (oxido de tris (1-(2 metil) aziridinil) fosfuro), y accion del mismo sobre Trypanosoma cruzi. Rev. Saúde públ., S. Paulo, 6:79-83, 1972.

tre los 3 grupos no hubo diferencia en cuanto al $\%$ de infección, las cifras correspondientes fueron: $83,3 \%, 84,0 \%$ y $84,2 \%$.

\section{DISCUS I ON}

Los resultados representados en las Figuras 2 y 3 sugieren que la esterilidad inducida quìmicamente en $R$. prolixus depende de tres factores fundamentales: la fertilidad intrínseca fisiológica de la hembra, el error del método y la susceptibilidad individual al esterilizante.

Analizando los valores de fertilidad que se obtuvieron en los testigos, se nota que en el grupo de las hembras hubo un individuo con $66,6 \%$ habiendo puesto sólo tres huevos, de los cuales eclosionaron dos. Esto sugiere que al estudiar la accion de un esterilizante, la fertilidad fisiológica de la hembra puede interferir alterando el efecto del producto.

Habiéndose trabajado con un microaplicador de mano, hay que tomar en cuenta el error del método: los valores extremos de las barras, que se salen de la "s", se podrían atribuir a que ese individuo recibió una dosis menor o mayor que la esperada.

La susceptibilidad individual incluiría los valores contenidos en el rango $\bar{x} \pm$ s. Este factor es el más interesante a considerar ya que permite conocer, en una población, el $\%$ de individuos menos susceptibles y la aparición de proble. mas de resistncia 7 .

Para poder estudiar la susceptibilidad individual, habría que reducir el error del método usando un microaplicador automático o empleando el producto químico marcado para poder medir la cantidad de sustancia suministrada.
Los resultados del metepa sobre $T$ cruzi indican que la dosis ensayada (100 $\mu \mathrm{g}$.), a pesar de esterilizar el vector, no afecta al parásito. Quizás dosis mayores podrian alterar el desarrollo de este último, pero como $200 \mu \mathrm{g}$. causam daños indeseables en el vector, no se creyó oportuno ensayar con dosis más altas.

Por otra parte, experimentos similares sobre parásitos 1 y virus 5 , han demostrado que estos son mucho más resistentes que los vectores a los esterilizantes químicos, concluyendo Bertram ${ }^{1}$ que, en vista de la acción mutágena de los agentes alkilantes, es preferible conformarse con solo matar o esterilizar los insectos usando pequeñas dosis y no exponer a un peligro mayor las otras formas de vida.

RSPSP-118

Feltciangeli, M. D. et al. - [Individual sterilization of Rhodnius prolixus Stal. by metepa (tris (1-(2 methiol) aziridinil) phosphoric oxide) and it action on Trypanossoma cruzi. Rev. Saúde públ., S. Paulo, 6:79-83, 1972.

Summary: The factors that interfere with the chemosterilization of $\mathbf{R}$. prolixus, when metepa is applied topically, are analysed. It is suggested that similar studies, based on individual fertility of insects, be periodically made to detect the appearance of resistance in triatominae populations. Data with indicates that metepa has no effect on $\mathrm{T}$. cruzi when $\mathrm{R}$. prolixus were infected with the parasite after chemosterilization, are prosented.

UnITERMS: Rhodnius prolixus*; Chemosterilization"; Metepa; Trypanossoma cruzi. 
FELICIANGELI, M. D. et al. - Esterilidad individual en Rhodnius prolixus Stal. tratados con metepa (oxido de tris (1-(2 metil) aziridinil) fosfuro), y accion del mismo sobre Trypanosoma cruzi. Rev. Saúde públ., S. Paulo, 6:79-83, 1972.

RSPSP-118

Feliciangeri, M. D. et al. - [Esterilidade induzida em Rhodnius prolixus. Stal. tratados com metepa (oxido de tris (1-(2 metil) aziridinil) fosforo) $e$ a ação do mesmo sobre Trypanosoma cruzi.] Rev. Saúde públ., $S$. Paulo, 6:79-83, 1972.

Resumo: Analisaram-se os fatores que intervêm na esterilidade induzida quimicamente em R. prolixus, mediante aplicações tópicas de metepa em várias doses. Sugere-se a realização de investigações mais profundas sobre a suscetibilidade individual, objetivando o conhecimento da composiçấo populacional em contato com o quimioesterilizante $e$ o provável aparecimento da resistência. Investigou-se também a ação de metepa sobre $\mathrm{T}$. cruzi em triatomineos infectados experimentalmente, com resultados negativos.

UNITERMOS: Rhodnius prolixus*; Quimiosterilização*; Metepa; Trypanossoma cruzi.

\section{AGRADECIMENTOS}

A los Drs J. C. Gómez Nuñez, J. W. Torrealba, J. Rabinovich; Jefe de la Sección de Estudios Biológicos de la División de Endemias Rurales, Jefe de la Cátedra de Parasitologia de la Universidad de Carabobo, Departamento de Ecología en el IVIC respectivamente, por sus valiosas observaciones.

\section{REFERENCIAS BIBLIOGRAFICAS}

1. BERTRAM, D. $\mathbf{S}$. et al. - Transmission of Plasmodium galinaceum Brumpt to chicks by Aedes aegypti sterilised by and alkilating agent, Thiotepa. J. trop. Med. Hyg., 67: 51-7, 1964.

2. DIAZ, F. - Tecnica do diagnostico na molestia de Chagas. Mem. Inst. Oswoaldo Cruz, 35: 335-42, 1940.

3. FELICIANGHLI, M. D. - Chemosterilization of adult Rhodnius prolixus by Metepa. 1970. (En prensa).

4. Gomes-NUNEZ, J. C. - Mass rearing of Rhodnius prolixus. Bull. Wld Elth Org., 31: 565-67, 1964.

5. KAPPUS, K. D. \& CORRISTAN, E. C. Effect of apholate and Metepa on Aedes aegypti infected with Venezuelan equine encephalomielitis virus. Amer. J. trop. Med. Hyg., 16: 599-43, 1967.

6. LINDQUIST. A. W. - Chemicals to sterilize insects. J. Wash. Acad. Sci. 51: 109, 1961 .

7. SACCA, G. et al. - Studio sperimentale di un ceppo di Musca domestica L., solezionato con il chemosterilante Metepa. Atti Soc. pelorit. Bci. fis. mat. nat., 12: 447-56, 1966.

8. TOPPOZADA, A. et al. - Chemosterilization of larvae and adults of the Egyptian cotton leawform, Prodenia litura by Apholate, Metepa and Tepa. J. econ. Ent., 59: 1125-28, 1966.

Recebido para publicạão cm 28-12-1971

Aprovado para publicagão em 7-1-1972 